\title{
AVERAGE RADIAL LIMITS IN WEIGHTED HARDY SPACES
}

\author{
ALEC MATHESON AND DAVID C. ULLRICH
}

\begin{abstract}
Weighted Hardy spaces are defined in the unit disc by specifying the rate of growth of $p$ th means near the boundary. Although a function in one of these spaces need have no radial limits, it is shown that in certain of these spaces "average radial limits" exist over an interval on the boundary. An integral representation in terms of these average radial limits is given, with an application to the question of existence of (pointwise) radial limits.
\end{abstract}

0. Introduction. Let $H_{\alpha}^{p}(0<p \leqslant \infty)$ denote the space of functions $f$ analytic in the unit disc $D \subseteq C$ satisfying the growth condition

$$
M_{p}(f, r) \leqslant c(1-r)^{-\alpha} \quad(0<r<1),
$$

where

$$
\begin{gathered}
M_{p}(f, r)=\left\{\frac{1}{2 \pi} \int_{-\pi}^{\pi}\left|f\left(r e^{i \theta}\right)\right|^{p} d \theta\right\}^{1 / p} \text { for } 0<p<\infty, \\
M_{\infty}(f, r)=\sup _{0 \leqslant \theta \leqslant 2 \pi}\left|f\left(r e^{i \theta}\right)\right|
\end{gathered}
$$

Defining

$$
\|f\|_{p, \alpha}=\sup _{0<r<1}(1-r)^{\alpha} M_{p}(f, r)
$$

gives a norm (if $p \geqslant 1$ ) on $H_{\alpha}^{p}$ making it into a Banach space (for completeness cf. [M]; for general background cf. [HL]).

For $f$ analytic in $D, t>0,0<\rho<1$ and $0 \leqslant \theta \leqslant 2 \pi$, we set

$$
A_{t}^{\rho} f\left(e^{i \theta}\right)=\frac{1}{2 t} \int_{-t}^{t} f\left(\rho e^{i(\theta-s)}\right) d s,
$$

and define

$$
A_{t} f\left(e^{i \theta}\right)=\lim _{\rho \rightarrow 1^{-}} A_{t}^{\rho} f\left(e^{i \theta}\right),
$$

provided the limit exists. If $A_{t} f\left(e^{i \theta}\right)$ exists, we call this an "average radial limit" of $f$ at the point $z=e^{i \theta}$.

The purpose of this note is to give in Theorem 1 and its corollary a precise description of those $H_{\alpha}^{p}$ spaces whose functions admit average radial limits at every boundary point. This is then applied to produce a theorem of Fatou type for these spaces.

Received by the editors July $21,1985$.

1980 Mathematics Subject Classification. Primary 31A20; Secondary 30D55, 31A10.

(C)1986 American Mathematical Society $0002-9939 / 86 \$ 1.00+\$ .25$ per page 
1. Existence of average radial limits. For $0<\beta \leqslant 1$, let $\Lambda_{\beta}$ denote the space of functions analytic in $D$, continuous in $\bar{D}$, and satisfying a Lipschitz condition of $\operatorname{order} \beta$ :

$$
\left|f\left(e^{i s}\right)-f\left(e^{i t}\right)\right| \leqslant c_{f}|s-t|^{\beta} .
$$

Theorem 1 will be an immediate consequence of the following theorem of Hardy and Littlewood (Theorem 5.1 in [D]):

THEOREM A. For $0<\beta \leqslant 1$ we have $f \in \Lambda_{\beta}$ if and only if $f^{\prime} \in H_{1-\beta}^{\infty}$; further

$$
c_{\beta}\|f\|_{\Lambda_{\beta}} \leqslant|f(0)|+\left\|f^{\prime}\right\|_{\infty, 1-\beta} \leqslant c_{\beta}^{\prime}\|f\|_{\Lambda_{\beta}} .
$$

TheOREM 1. If $f \in H_{\alpha}^{\infty}, \alpha<1$, then $A_{t} f\left(e^{i \theta}\right)$ exists for $0<t \leqslant \pi, 0 \leqslant \theta \leqslant 2 \pi$; also

$$
\left|A_{t} f\left(e^{i \theta}\right)\right| \leqslant c_{\alpha}\left(\|f\|_{\infty, \alpha} / t^{\alpha}\right) .
$$

Proof. Let $f \in H_{\alpha}^{\infty}$. We may suppose $\theta=0$ and $f(0)=0$. Choose $F$ analytic in $D$ so that $F^{\prime}(z)=f(z) / z$. Note that $f(z) / z \in H_{\alpha}^{\infty}$, so Theorem A implies $F \in$ $\Lambda_{1-\alpha}$. Now

$$
\begin{aligned}
A_{t}^{\rho} f(1) & =\frac{1}{2 t i} \int_{-t}^{t} \frac{f\left(\rho e^{i \theta}\right)}{\rho e^{i \theta}} i \rho e^{i \theta} d \theta \\
& =\frac{1}{2 t i} \int_{\rho e^{-i t}}^{\rho e^{i t}} \frac{f(z)}{z} d z=\frac{1}{2 t i}\left(F\left(\rho e^{i t}\right)-F\left(\rho e^{-i t}\right)\right),
\end{aligned}
$$

so $A_{t} f(1)=(1 / 2 t i)\left(F\left(e^{i t}\right)-F\left(e^{-i t}\right)\right)$ exists and

$$
\left|A_{t} f(1)\right| \leqslant(1 / 2 t) t^{1-\alpha}\|F\|_{\Lambda_{1-\alpha}} \leqslant c_{\alpha}\left(\|f\|_{\infty, \alpha} / t^{\alpha}\right) .
$$

This completes the proof.

Corollary. If $f \in H_{\alpha}^{p}, \alpha<1-1 / p$, then $A_{t} f\left(e^{i \theta}\right)$ exists and

$$
\left|A_{t} f\left(e^{i \theta}\right)\right| \leqslant c_{\alpha, p}\left(\|f\|_{p, \alpha / t^{\alpha+1 / p}}\right) .
$$

Proof. Another theorem of Hardy and Littlewood (Theorem 5.9 in [D]) states that $H_{\alpha}^{p} \subseteq H_{\alpha+1 / p}^{\infty}$; the corollary follows.

This corollary is sharp in the following sense: Let $f(z)=(1-z)^{-1}$. Then $f \in H_{\alpha}^{p}$ whenever $\alpha \geqslant 1-1 / p(p>1)$ although $A_{t} f\left(e^{i t}\right)$ fails to exist. (In $\$ 3$ we will describe an $f \in H_{1}^{\infty}$ such that $A_{t} f\left(e^{i \theta}\right)$ exists for no value of $\theta$.)

2. A Fatou theorem. We now show how $f(r)(0 \leqslant r<1)$ can be recovered from $\left\{A_{t} f(1): 0<t \leqslant \pi\right\}$ by an "integrated Poisson formula":

Let $P_{r}(\theta)=\left(1-r^{2}\right) /\left(1-2 r \cos (\theta)+r^{2}\right)$ be the Poisson kernel and let $P_{r}^{\prime}(\theta)=$ $(\partial / \partial \theta) P_{r}(\theta)$. Note that $P_{r}^{\prime} \leqslant 0$ on $[0, \pi]$. For $0 \leqslant r<1$ define a measure $\mu_{r}$ on $[0, \pi]$ by

$$
\int_{0}^{\pi} \phi(t) d \mu_{r}(t)=\frac{1-r}{1+r} \phi(\pi)-\frac{1}{\pi} \int_{0}^{\pi} t P_{r}^{\prime}(t) \phi(t) d t .
$$


The following lemmas are each proved by integration by parts.

LEMMA 1. The measures $\mu_{r}$ satisfy

(a) $\mu_{r} \geqslant 0$,

(b) $\mu_{r}([0, \pi])=1$,

(c) for any $\delta>0, \lim _{r \rightarrow 1} \mu_{r}([\delta, \pi])=0$.

LEMMA 2. Let $f \in L^{1}(T)$ and $A_{t} f\left(e^{i \theta}\right)=(1 / 2 t) \int_{-t}^{t} f\left(e^{i(\theta-s)}\right) d s$. Then

$$
P[f]\left(r e^{i \theta}\right)=\int_{0}^{\pi} A_{t} f\left(e^{i \theta}\right) d \mu_{r}(t) \quad(0 \leqslant r<1) .
$$

( Here $P[f]\left(r e^{i \theta}\right)=(1 / 2 \pi) \int_{-\pi}^{\pi} f\left(e^{i(\theta-s)}\right) P_{r}(s) d s$, as usual.)

THEOREM 2. If $f \in H_{\alpha}^{\infty}, \alpha<1$, then

$$
f\left(r e^{i \theta}\right)=\int_{0}^{\pi} A_{t} f\left(e^{i \theta}\right) d \mu_{r}(t) \quad\left(r e^{i \theta} \in D\right) .
$$

Proof. Let $0<p<1$ and $f_{\rho}(z)=f(\rho z)$. Lemma 2 shows that

$$
f_{\rho}\left(r e^{i \theta}\right)=\int_{0}^{\pi} A_{t} f_{\rho}\left(e^{i \theta}\right) d \mu_{r}(t) .
$$

But $A_{t} f_{\rho}=A_{t}^{\rho} f \rightarrow A_{t} f$ as $\rho \rightarrow 1$, and for all $\rho$ we have

$$
\left|A_{t} f_{\rho}\right| \leqslant c_{\alpha}\left(\left\|f_{\rho}\right\|_{\infty, \alpha} / t^{\alpha}\right) \leqslant c_{\alpha}\left(\|f\|_{\infty, \alpha} / t^{\alpha}\right) \text {. }
$$

Since $\int_{0}^{\pi} t^{-\alpha} d \mu_{r}(t)<\infty$, the theorem follows by dominated convergence.

Corollary. Suppose $f \in H_{\alpha}^{\infty}, \alpha<1$. If $\lim _{t \rightarrow 0^{+}} A_{t} f\left(e^{i \theta}\right)=0$, then $\lim _{r \rightarrow 1^{-}} f\left(r e^{i \theta}\right)=0$.

\section{Proof. Lemma 1.}

The converse of this corollary is false: For any $p<\infty$ there exists $f \in H^{p}=H_{0}^{p}$ $\subseteq H_{1 / p}^{\infty}$ such that $\lim _{r \rightarrow 1^{-}} f(r)=0$, but $\lim _{t \rightarrow 0^{+}} A_{t} f(1)$ does not exist. If, on the other hand, $f \in H^{\infty}$, then a theorem of Loomis [L] states that $\lim _{r \rightarrow 1^{-}} f(r)=0$ implies $\lim _{t \rightarrow 0^{+}} A_{t} f(1)=0$. This last statement has recently been extended to $f$ in the Bloch space; see [U].

3. An example. The example mentioned at the end of $\$ 1$ leaves open the possibility that, e.g., $A_{t} f\left(e^{i \theta}\right)$ should exist for almost all $\theta$ if $f \in H_{1}^{\infty}$. This is not the case:

EXAMPLE. Let $0<t<\pi$. There exists $f \in H_{1}^{\infty}$ such that $A_{t} f\left(e^{i \theta}\right)$ exists for no value of $\theta$.

Proof. Fix $t \in(0, \pi)$. Let $S=\{n=1,2, \ldots:|\sin (n t)| \geqslant 1 / 2\}$. Note that $S$ is infinite, since $0<t<\pi$. For $n=0,1, \ldots$ let $e_{n}(z)=z^{n}$; note that for $n \in S$,

$$
\left|e_{n}\left(e^{i t} z\right)-e_{n}\left(e^{-i t} z\right)\right| \geqslant|z|^{n} \text {. }
$$

Suppose $n_{1}, \ldots, n_{N} \in S$ and $0<r_{1}<r_{1}^{\prime}<\cdots<r_{N}<r_{N}^{\prime}<1$ have been chosen so that if $F_{N}=\sum_{j=1}^{N} e_{n_{j}}$ and $G_{N}(z)=F_{N}\left(e^{i t} z\right)-F_{N}\left(e^{-i t} z\right)$, then

$$
(1-|z|)\left|F_{N}^{\prime}(z)\right|<1 \quad(z \in D)
$$

and

$$
\left|G_{N}\left(r_{j} e^{i \theta}\right)-G_{N}\left(r_{j}^{\prime} e^{i \theta}\right)\right|>1 / 2 \quad(j=1,2, \ldots, N ; 0 \leqslant \theta \leqslant 2 \pi) .
$$


Now if $n_{N+1} \in S$ is large enough, (2) holds with $N+1$ in place of $N$. It follows from (1) that if $n_{N+1} \in S$ is large enough, then we may choose $r_{N+1}$ and $r_{N+1}^{\prime}$, with $r_{N}^{\prime}<r_{N+1}<r_{N+1}^{\prime}<1$, such that (3) holds with $N+1$ in place of $N$.

Let $F=\lim _{N \rightarrow \infty} F_{N}, G=\lim _{N \rightarrow \infty} G_{N}$, so $G(z)=F\left(e^{i t} z\right)-F\left(e^{-i t} z\right)$. Let $f(z)$ $=z F^{\prime}(z)$. Then (2) implies that $f \in H_{1}^{\infty}$. On the other hand, the proof of Theorem 1 shows that

$$
\left|A_{i}^{r_{j}} f\left(e^{i \theta}\right)-A_{i}^{r_{j}^{\prime}} f\left(e^{i \theta}\right)\right|=(1 / 2 t)\left|G\left(r_{j} e^{i \theta}\right)-G\left(r_{j}^{\prime} e^{i \theta}\right)\right| \geqslant 1 / 4 t,
$$

so $\lim _{r \rightarrow 1} A_{t}^{r} f\left(e^{i \theta}\right)$ does not exist.

\section{REFERENCES}

[D] P. L. Duren, Theory of $H^{p}$ spaces, Academic Press, New York, 1970.

[HL] G. H. Hardy and J. E. Littlewood, Theorems concerning mean values of analytic or harmonic functions, Quart. J. Math. Oxford Ser. 8 (1937), 161-171.

[L] L. H. Loomis, The converse of the Fatou theorem for positive harmonic functions, Trans. Amer. Math. Soc. 53 (1943), 239.

[M] A. Matheson, A multiplier theorem for analytic functions of slow mean growth, Proc. Amer. Math. Soc. 77 (1979), 53-57.

[U] D. C. Ullrich, Radial limits of Bloch functions in the unit disc, Bull. London Math. Soc. 18 (1986).

Department of Mathematics, Lamar University, Beaumont, Texas 77710

Department of Mathematics, Oklahoma State University, Stillwater, Oklahoma 74078 